\title{
SCANNING ELECTRON AND OPTICAL MICROSCOPIC STUDIES OF THE SYSTEM OF PORCINE ZONULAR FIBRES
}

\section{Zoltán Imre Bocskai ${ }^{1}$, Zoltán Kiss² ${ }^{2}$ Gábor László Sándor ${ }^{3}$, Imre Bojtár ${ }^{1}$, Zoltán Zsolt Nagy ${ }^{4}$}

${ }^{1}$ Budapest University of Technology and Economics, Faculty of Civil Engineering, Department of Structural Mechanics

${ }^{2}$ Budapest University of Technology and Economics, Faculty of Mechanical Engineering, Department of Polymer Engineering

${ }^{3}$ Semmelweis University, Department of Ophthalmology

${ }^{4}$ Semmelweis University, Department of Ophthalmology and Faculty of Health Sciences

zbocskai@mail.bme.hu

\begin{abstract}
The mechanical behaviour of the zonular apparatus greatly affects the accommodation process because it moves out from the ciliary muscle and runs into the lens capsule. The zonular fibres convey the force between the ciliary body and the crystalline lens. For the measurement of the mechanical properties of zonular fibres is necessary to know its geometry. In this paper we analyzed the network of porcine zonular fibres with scanning electron and optical microscope. We manifested that the porcine zonular fibre system differs from human and monkey eyes. The thickness of the apparatus of intertwined zonular fibres is approximately $45 \mu \mathrm{m}$ and the length from the origin to the insertion is about $1.5 \mathrm{~mm}$. Based on these informations the input parameters of numerical modelling can be more precise to clarify the accommodation process.
\end{abstract}

Keywords: zonular fibres, bundles, scanning electron microscope

\section{Introduction}

The zonular fibres play a major role in the mechanism of accommodation. The force from the ciliary body is transferring to the lens through the zonular fibre system. ${ }^{1}$ Based on the Helmholtz theory ${ }^{1}$ the geometry of the apparatus of the zonules is very important at the accommodation process of the eye. For measuring of the mechanical properties of these fibres - for example with tension tests - we need the geometry e.g. thickness and length parameters of the bundles. Many papers have been dealt with the analysis of zonules usually with electron microscope. ${ }^{2-6}$ For the investigation of the mechanical behaviour of zonular fibres in macroscopic level first of all we need the geometry of the network system. There are only very few data about the size of the zonular bundles. Our purpose in this paper is to investigate the bounding dimension of the zonular group with three different methods. We used scanning electron microscope for the first method and optical microscope for the second and the third one. The network of the fibre system was not considered in details.

\section{Materials and Methods}

The porcine eye samples were harvested from a local slaughterhouse. We obtained the globes of the eyes (bulbus oculi) from 6-7 months old, weighing $100-120 \mathrm{~kg}$, male animals. The transport time from the slaughterhouse to the laboratory was one and the half hours. The transport temperature was +4 degree of Celsius and 
it was held with cooler bag. 20 eyeballs were included in this study. 5 eyeballs were used for the scanning electron microscope analysis and 10 (5-5) for the optical microscope analysis. The remaining globes with obvious deformation or damage were excluded. The network and the thickness of the zonular group were analyzed in three different ways. In every case we took care about the integrity of the zonular network, when we removed - with scissors and forceps - the posterior side with the aqueous humour and the hyaloid membrane. After this step the cornea has been removed at the limbus and finally the iris. Samples were prepared and analyzed as quickly as possible to avoid any biological decomposition.

\subsection{Measuring with scanning electron microscope (SEM)}

The specimens were fixed for scanning electron microscopy in $8 \%$ paraformaldehyde. The fixed samples were dehydrated in a series of ethanol dilutions (20\%-96\% vol/vol), fol-
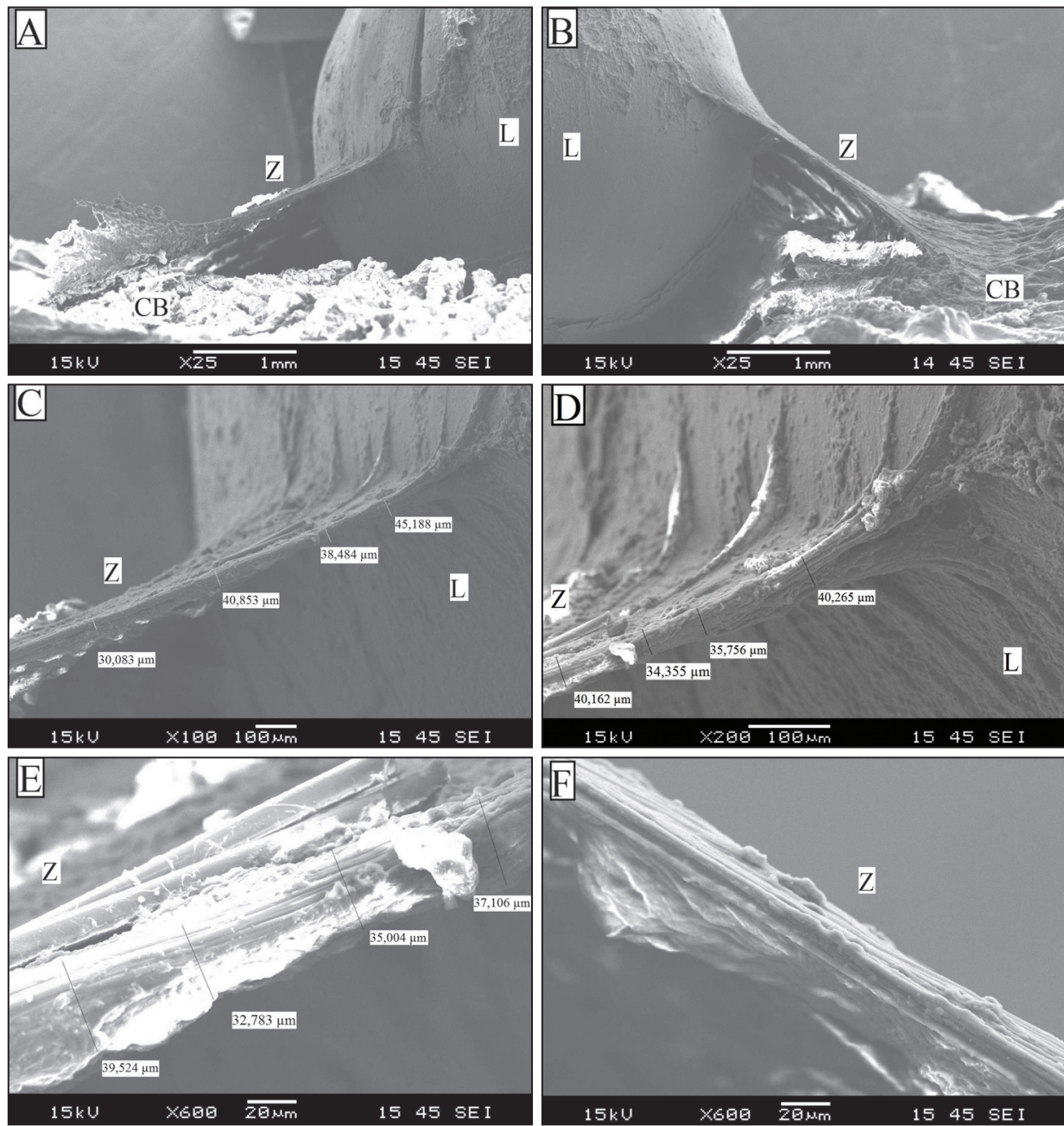

Figure 1. SEM images about the zonular bundles L: lens; Z: zonules; CB: ciliary body 
lowed by vacuum-drying. The specimens were mounted on double-sided carbon tape, sputter coated with gold and images were taken at various magnifications with scanning electron microscope JSM 6380LA (JEOL Ltd., Tokyo, Japan) and with the manufacturer's software. Figure 1 shows some of the SEM images.

\subsection{Measuring with optical microscope (OM I. and OM II.)}

We would like to measure the average thickness dimension of the zonular group as we stated in the introduction part thus we analyzed the samples with optical microscope also (Olympus BX 51M, Olympus Corporation, Tokyo, Japan) with two different methods.

In the first way the eyeballs were slightly fixed in $8 \%$ formaldehyde solution for 24 hours. After this step the zonular fibres with the crystalline lens in the slightly fixed position were prepared because in the eyeball the intraocular pressure causes certain amount of tensile strength in the fibres despite the fact that after the death the state of rigor mortis occurred during 2-4 hours. In this position we made a section toward the zonules, the lens and the ciliary body. With this method we got a sample what could help to analyse the thickness of the zonular apparatus with optical microscope (OM I.). Figure 2 shows some of the images and the measured thickness values.

In the second way of optical microscope examination (OM II.) we cut the posterior part behind the lens (sclera, choroid, retina and vitreous body) and the anterior side with the cornea and iris from the fresh eyeballs. The hyaloid membrane was removed very carefully. Thus we have the intact ( 2 hours delayed from the slaughtering) anterior side with the lens, zonules and ciliary body with the narrow stripe of sclera without any fixative to preserve a native in situ architecture. These prepared specimens were filled with polyester resin. We waited until the resin become solid and then made a section across the lens, zonules and ciliary muscle. After these steps the specimens were polished with Struers (Struers $\mathrm{GmbH}$, Germany) automatic polishing machine then we could analyse the surface of the section with the optical microscope. With this method the thickness of the group of intact zonular fibres was measurable - without any dehydration according to the gap between the solid layers of resins (green and black layers in Figure 3). Some of the images and the measured thickness values are in Figure 3.
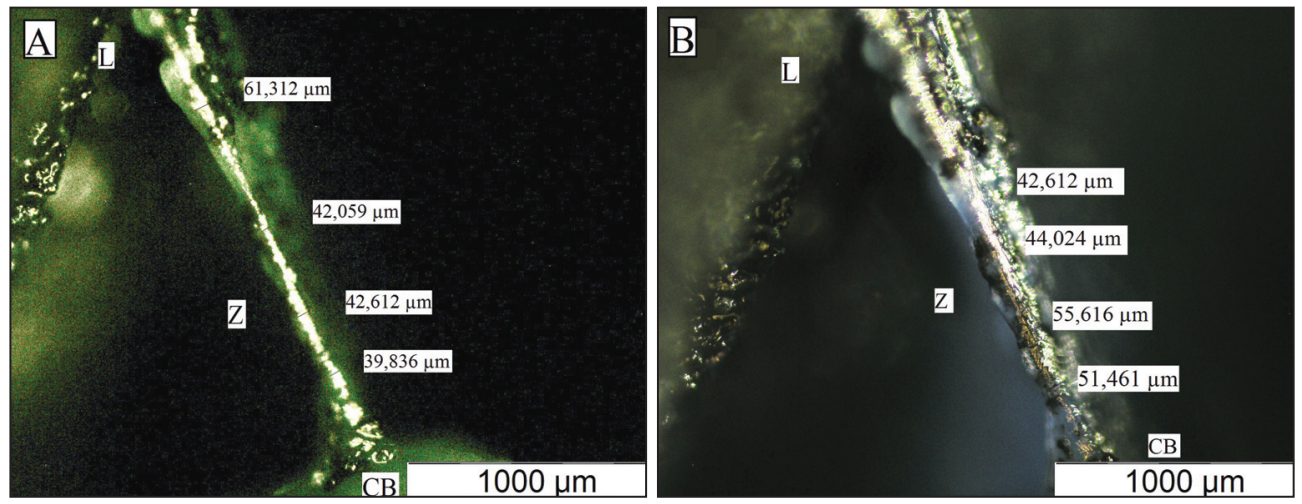

Figure 2. Optical microscope images about the zonular bundles L: lens;

$\mathrm{Z}$ : zonules; CB: ciliary body 


\section{Results}

The thin filaments run up to the equatorial edge from the interlacing zonular bundles (Figures $1 / D$ and $4 / A)$. In Figure $1 / A-B$ and in Figure $4 / B$ we can see that the zonular filaments attached to the bottom of the basis of ciliary body valleys. Near the equatorial connections the zonular bundles become more organised and approximately parallel as you can see in Figure $1 / E-F$ until the origin from the ciliary valleys. This roughly parallel structure of the
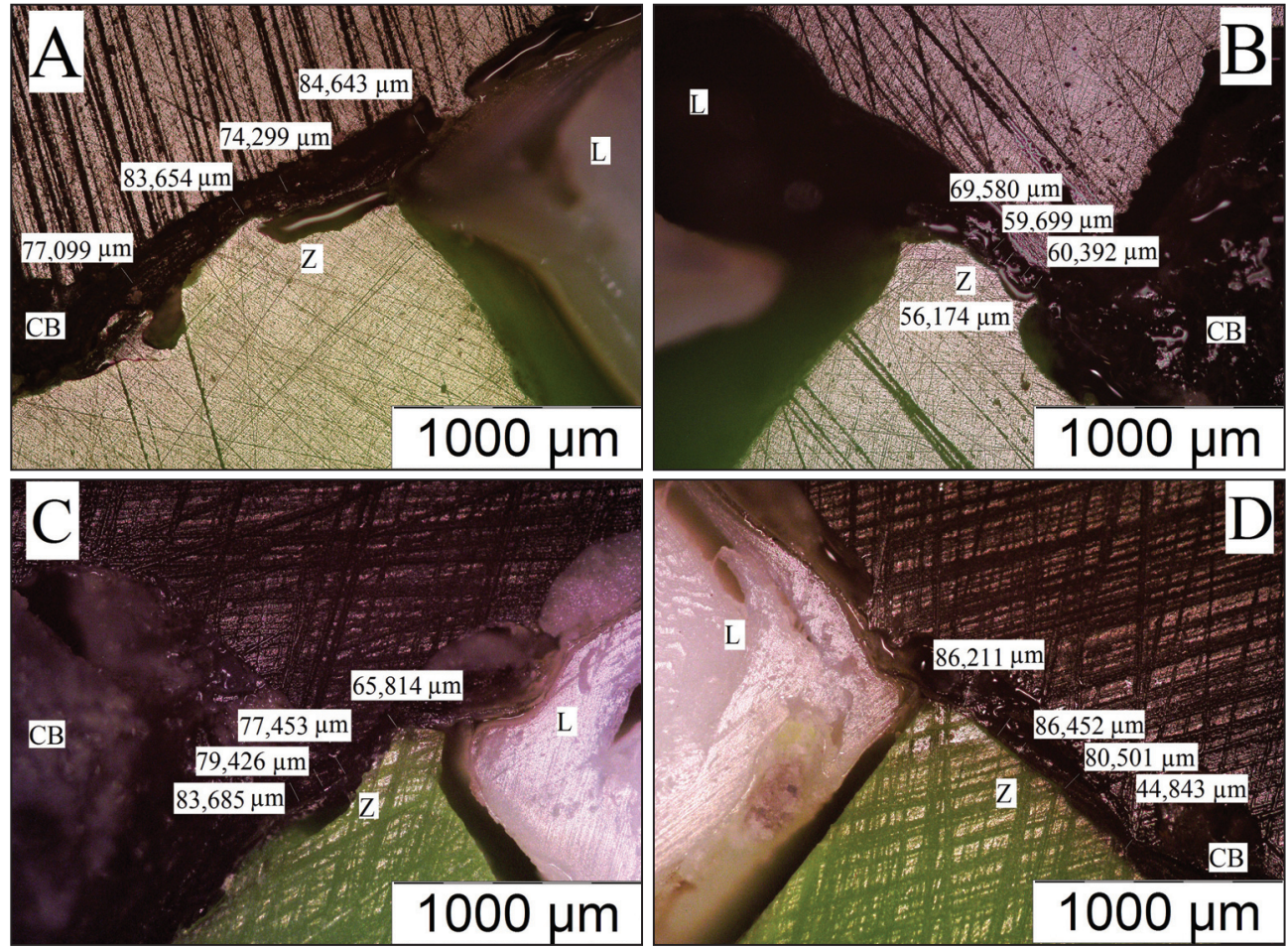

Figure 3. Optical microscope images about the zonular fibres in the resin cast L: lens; Z: zonules; CB: ciliary body; black: anterior side; green: posterior side
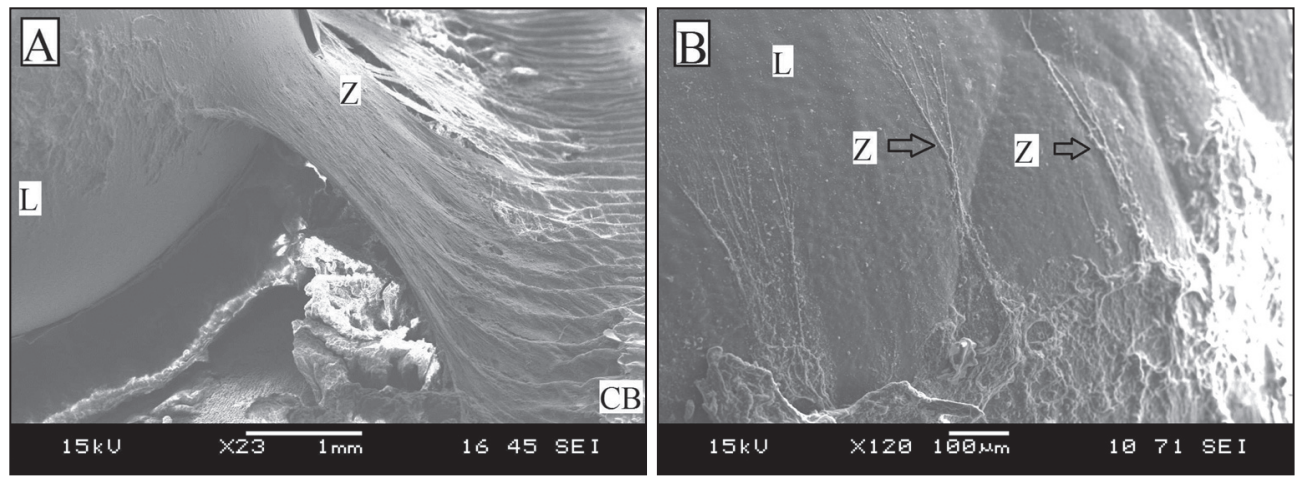

Figure 4. SEM images about the origin and insertion of the zonular bundles L: lens, Z: zonules 

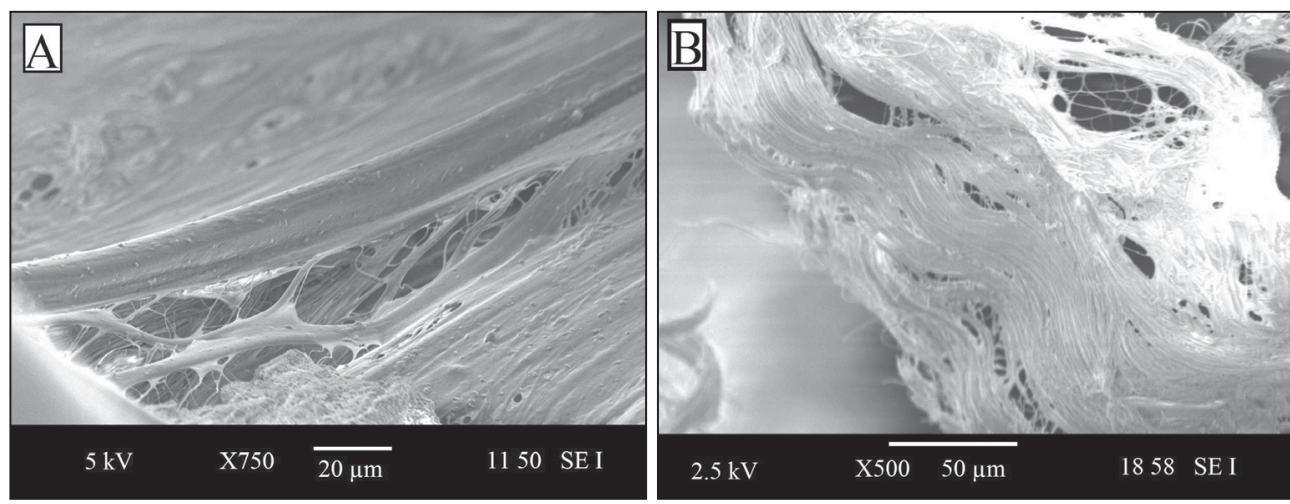

Figure 5. SEM images about the filaments and their interlacing

zonular network is permanent between the start and the end areas (Figure $1 / A-C$ ). The interlocking collateral layout is more conspicuous in higher magnification as shown in Figure 5.

Figure 6 shows the evidence of the tension force - despite of the rigor mortis - in the zonular fibres. According to the fact that during the preparation for SEM measurements at few samples the zonules injured or torn due to dehydration. As you can see in Figure 6 the filaments after the disruption became curled because fundamentally tensile force loaded them.

The thickness of the zonular apparatus of each specimen was measured in four different positions where the bundles were close to constant size and parallel as we mentioned before.

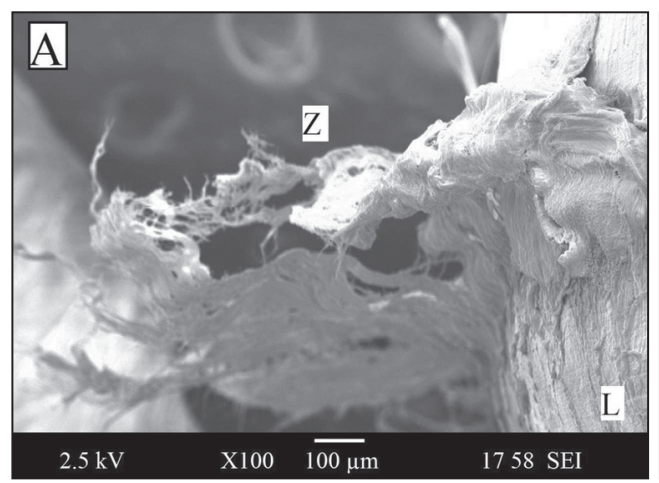

We measured the lowest average thickness val-

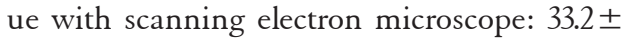
$\pm 9.1 \mu \mathrm{m}$. With the second method (OM I.) the average thickness was a bit wider: $43.2 \pm 9.6 \mu \mathrm{m}$. The third method (OM II.) resulted in an average $73.1 \pm 12.5 \mu \mathrm{m}$ thickness for the bundles. Figure 7 shows the mean thickness values and the standard deviations and the minimum/ maximum values based on the three different methods.

We also measured the length of the bundles from the ciliary muscle to the lens capsule. According to the SEM images we have an average $2.18 \pm 0.25 \mathrm{~mm}$ length for the bundles. The optical microscope analyses showed 1.92 \pm $\pm 0.05 \mathrm{~mm}$ and $1.18 \pm 0.37 \mathrm{~mm}$ average length value (OM I. and OM II.). Figure 7 also shows

Figure 6. SEM images about the torn fibres, side view and front view L: lens; Z: zonules

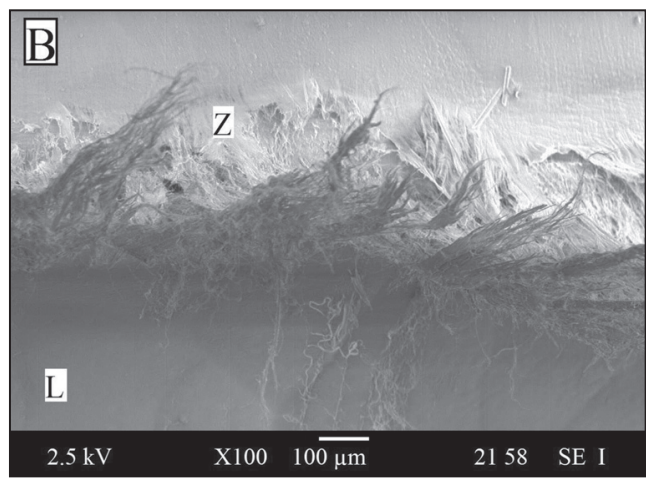



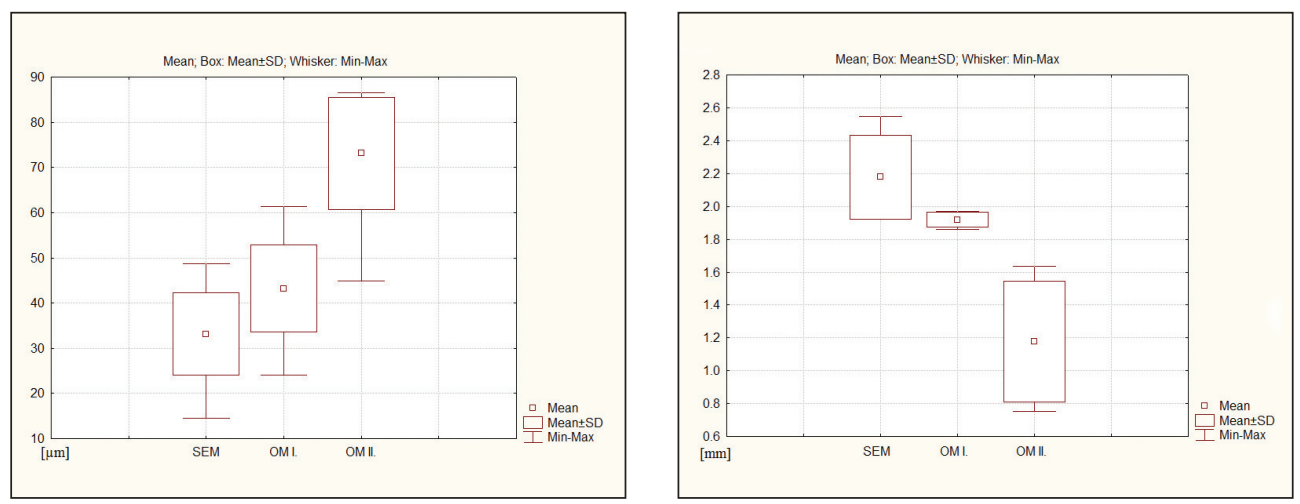

Figure 7. Results about the thickness and the length of the bundles (SEM, OM I., OM II.)

the results about the length of the zonular bundles from the three different methods in a statistical way.

\section{Discussion}

We can state that - based on the scanning electron microscope images - the network of the zonular fibres differs from the human and monkey zonules. ${ }^{2,5}$ As shown in Figure 1 it is obvious that the zonular network only connected at the equatorial stripe of the lens. We can see in Figure 1/D that the zonular fibres spread at the equatorial line of the lens but their structure is clearly distinguishable from the lens capsule.

We also concluded the average thickness of the porcine zonular bundles with different methods. The thickness was noticeably larger with OM II. method than with SEM or OM I. methods. This result was expected because the samples for SEM were almost completely dehydrated and the specimens for OM II. had natural moister content. The OM I. method resulted in the thickness values between the two other methods because in this case the moisture content was also between the other two hence these results served a control measurement.
All of the used methods have advantages and disadvantages (e.g. hydrated and dehydrated conditions, fixed and non-fixed states) but according to our opinion these techniques are reliable to estimate the minimum and the maximum range of the thickness of the bundles.

The average thickness values are comparable with reference ${ }^{7}$ who measured the thickness values of bovine zonular apparatus. They have been reported $45-55 \mu \mathrm{m}$ thickness but it should be noted that they used digestive enzyme (hyaluronidase) during their preparation technique.

The length measured by OM II. method was a bit shorter than the length with the other two methods because at SEM and OM I. analysis the ciliary muscle of the samples was in slightly different position. We saw longer length at SEM and OM I. because the crests of the ciliary process were in lower position thus the whole length of the zonular fibres was visible according to their origin from the bottom of the ciliary valleys.

Based on our analyses we can state that the geometry of the zonular bundles is given thus we can measure the mechanical parameters in easier way and we can refine the theory of the dynamic accommodation mechanism. 


\section{REFERENCES}

1. Bocskai Z, Bojtár I. Biomechanical modelling of the accommodation problem of human eye. Periodica Polytechnica Civil Engineering 2013; 57(1):1-7.

2. Bernal A, Parel JM, Manns F. Evidence for posterior zonular fiber attachment on the anterior hyaloid membrane. Investigative Ophthalmology \& Visual Science 2006;47:4708-13.

3. Curtis $R$. The suspensory apparatus of the canine lens. Journal of Anatomy 1983;136:69-83.

4. Farnsworth PN, Mauriello JA, Burke-Gadomski P Kulyk T, Cinotti AA. Surface ultrastructure of the human lens capsule and zonular attachments. Investigative Ophthalmology 1976;15:36-40.
5. Rohen JW. Scanning electron microscopic studies of the zonular apparatus in human and monkey eyes. Investigative Ophthalmology \& Visual Science 1979;18:133-44.

6. Streeten $B W$. The zonular insertion: a scanning electron microscopic study. Investigative Ophthalmology \& Visual Science 1977;16:364-75.

7. Wright DM, Duance VC, Wess TJ, Kielty CM, Purslow PP. The supramolecular organisation of fibrillin-rich microfibrils determines the mechanical properties of bovine zonular filaments. The Journal of Experimental Biology 1999;202: 3011-20.

The work reported in the paper has been developed in the framework of the project „Talent care and cultivation in the scientific workshops of BME” project. This project is supported by the grant TÁMOP-4.2.2.B-10/1-2010-0009.

\section{Zoltán Imre Bocskai}

Budapest University of Technology and Economics, Faculty of Civil Engineering H-1111 Budapest, Múegyetem rkp. 3. K ép. mf 63.

Tel.: (+36) $1463-1434$ 